\title{
Progenitor neutron stars of the lightest and heaviest millisecond pulsars
}

\author{
M. Fortin ${ }^{1,2,3}$, M. Bejger ${ }^{1}$, P. Haensel ${ }^{1}$, and J. L. Zdunik ${ }^{1}$ \\ ${ }^{1}$ N. Copernicus Astronomical Center, Polish Academy of Sciences, Bartycka 18, 00-716 Warszawa, Poland \\ e-mail: [fortin; bejger; haensel; jlz]@camk.edu.pl \\ 2 LUTh, UMR 8102 du CNRS, Observatoire de Paris, 92195 Meudon Cedex, France \\ 3 Istituto Nazionale di Fisica Nucleare - Sezione di Roma, P.le Aldo Moro 2, 00185 Roma, Italy
}

Received 3 September 2014 / Accepted 12 November 2015

\begin{abstract}
Context. The recent mass measurements of two binary millisecond pulsars, PSR J1614-2230 and PSR J0751+1807 with a mass $M=1.97 \pm 0.04 M_{\odot}$ and $M=1.26 \pm 0.14 M_{\odot}$, respectively, indicate a wide range of masses for such objects and possibly also a broad spectrum of masses of neutron stars born in core-collapse supernovae.

Aims. Starting from the zero-age main sequence binary stage, we aim at inferring the birth masses of PSR J1614-2230 and PSR J0751+1807 by taking the differences in the evolutionary stages preceding their formation into account.

Methods. Using simulations for the evolution of binary stars, we reconstruct the evolutionary tracks leading to the formation of PSR J1614-2230 and PSR J0751+1807. We analyse in detail the spin evolution due to the accretion of matter from a disk in the intermediate-mass/low-mass X-ray binary. We consider two equations of state of dense matter, one for purely nucleonic matter and the other one including a high-density softening due to the appearance of hyperons. Stationary and axisymmetric stellar configurations in general relativity are used, together with a recent magnetic torque model and observationally-motivated laws for the decay of magnetic field.

Results. The estimated birth mass of the neutron stars PSR J0751+1807 and PSR J1614-2230 could be as low as $1.0 M_{\odot}$ and as high as $1.9 M_{\odot}$, respectively. These values depend weakly on the equation of state and the assumed model for the magnetic field and its accretion-induced decay.

Conclusions. The masses of progenitor neutron stars of recycled pulsars span a broad interval from $1.0 M_{\odot}$ to $1.9 M_{\odot}$. Including the effect of a slow Roche-lobe detachment phase, which could be relevant for PSR J0751+1807, would make the lower mass limit even lower. A realistic theory for core-collapse supernovæ should account for this wide range of mass.
\end{abstract}

Key words. equation of state - stars: neutron - accretion, accretion disks - dense matter

\section{Introduction}

Millisecond radio pulsars (defined here as those with a spin period $P<10 \mathrm{~ms}$ ) have several unique properties that make them very interesting objects to study, both observationally and theoretically. They are the most rapid stellar rotators with a spin frequency $f=1 / P$ up to $716 \mathrm{~Hz}$ (Hessels et al. 2006). Their spin periods are extremely stable with a typical period increase, owing to the spin angular momentum loss associated with magnetodipole radiation: $\dot{P} \sim 10^{-20} \mathrm{~s} \mathrm{~s}^{-1} \sim 10^{-13} \mathrm{~s} \mathrm{yr}^{-1}$. Consequently, their magnetic field $B$, as estimated from the timing properties, are three to four orders of magnitude weaker than those of normal radio pulsars, for which $B \simeq 10^{12} \mathrm{G}$.

According to the current theory of neutron star (NS) evolution, millisecond (radio) pulsars (MSPs) originate in "radiodead" pulsars via the accretion-caused spin-up in low-mass X-ray binaries (LMXBs, see Alpar et al. 1982; Radhakrishnan \& Srinivasan 1982). During this "recycling" process, the rotation frequency increases from an initial value $\lesssim 0.1 \mathrm{~Hz}$ to a final $\sim 500 \mathrm{~Hz}$ in $\sim 10^{8}-10^{9} \mathrm{yr}$. The process is associated with the accretion of matter via an accretion disk around the NS. Millisecond X-ray pulsars become millisecond radio pulsars after the accretion process stops. This scenario has been corroborated by the detection of millisecond X-ray pulsations in LMXBs, interpreted as the manifestation of rotating and accreting NSs (Wijnands \& van der Klis 1998) and the observations of three objects in transition from a state of accretion with X-ray emission to a rotation-powered state with radio emission and/or vice versa: PSR J1023+0038 (Archibald et al. 2009; Patruno et al. 2014), PSR J1824-2452I (Papitto et al. 2013) and XSS J12270-4859 (Bassa et al. 2014).

The difference of typically three to four orders of magnitude in the magnetic field strength of MSPs and normal pulsars is explained either by the "burying" of the original magnetic field under a layer of accreted material (Bisnovatyi-Kogan \& Komberg 1971; Taam \& van den Heuvel 1986; Cumming et al. 2001) and/or by the Ohmic dissipation of electric currents in the accretion-heated crust (Romani 1990; Geppert \& Urpin 1994).

It is expected that the "recycling" process in LMXBs is a particularly widespread mechanism in dense stellar systems such as globular clusters. This is in accordance with the peculiar structure of the MSP population (Lorimer 2008): out of a total of about 260 MSPs, $\sim 60 \%$ are in binaries, whereas for other (nonmillisecond) pulsars, this percentage is one order of magnitude lower ( 4\%). Simultaneously, about half (117) of all MSPs are located in 23 Galactic globular clusters. Finally, some $50 \%$ of the 
Table 1. Measured parameters of the binary pulsars PSR J1614-2230 and PSR J0751+1807 and the masses of their WD companions.

\begin{tabular}{ccccccccc}
\hline \hline PSR & $\begin{array}{c}M_{\text {PSR }} \\
\left(M_{\odot}\right)\end{array}$ & $\begin{array}{c}P \\
(\mathrm{~ms})\end{array}$ & $\begin{array}{c}f \\
(\mathrm{~Hz})\end{array}$ & $\begin{array}{c}\dot{P} \\
\left(10^{-21}\right)\end{array}$ & $P_{\text {orb }}$ & $e$ & $\begin{array}{c}M_{\mathrm{WD}} \\
\left(M_{\odot}\right)\end{array}$ & $\begin{array}{c}B_{0} \\
\left(10^{8} \mathrm{G}\right)\end{array}$ \\
\hline $\mathrm{J} 1614-2230$ & $1.97 \pm 0.04$ & 3.15 & 317 & 9.6 & $8.7 \mathrm{~d}$ & $1.3 \times 10^{-6}$ & $0.500 \pm 0.006$ & 1.76 \\
$\mathrm{~J} 0751+1807$ & $1.26 \pm 0.14$ & 3.48 & 287 & 7.8 & $6.3 \mathrm{~h}$ & $7.1 \times 10^{-7}$ & $0.12 \pm 0.02$ & 1.66 \\
\hline
\end{tabular}

Notes. $B_{0}$ is the canonical value of the magnetic field obtained using the dipole formula Eq. (3) for an ortogonal rotator and the "canonical" NS radius and the moment of inertia: $R_{0}=10 \mathrm{~km}$ and $I_{0}=10^{45} \mathrm{~g} \mathrm{~cm}^{2}$.

globular cluster MSPs are found in binary systems ${ }^{1}$ (Manchester et al. 2005).

The widely accepted recycling mechanism in LMXBs suggests that rapid MSPs (say those with $P<5 \mathrm{~ms}$ ) are likely to be massive. Therefore, they are important for the observational determination of the maximum allowable mass for NSs. This upper bound is a crucial constraint on the poorly known equation of state (EOS) at supra-nuclear density. The precise measurement of the mass $M=1.67 \pm 0.02 M_{\odot}$ of PSR J1903+0327 (Freire et al. 2011) and, even more so, of $1.97 \pm 0.04 M_{\odot}$ for PSR J1614-2230 (Demorest et al. 2010) confirms that the population of MSPs contains massive NSs. The most massive pulsar to date is PSR J0348+0432 with $M=2.01 \pm 0.04 M_{\odot}$ (Antoniadis et al. 2013). Its properties are a relatively long spin period $(P=39 \mathrm{~ms})$ and a short orbital period $\left(P_{\mathrm{orb}}=2.46 \mathrm{~h}\right)$ for a recycled pulsar. Combined with the low mass of its helium white-dwarf (WD) companion, $M_{\mathrm{WD}}=0.17 M_{\odot}$, the case of PSR J0348+0432 is challenging for stellar evolution theory (see e.g. Antoniadis et al. 2013), and its formation will not be addressed in the following. On the other side of the mass spectrum, PSR J0751+1807 only has a mass of $1.26 \pm 0.14 M_{\odot}$ (Nice et al. 2008; all measurements are given at $1 \sigma$ confidence level). As of today, the masses of MSPs are therefore bracketed by $1.26 \pm 0.14 M_{\odot}$ and $1.97 \pm 0.04 M_{\odot}$. We focus on PSR J1614-2230 and PSR J0751+1807, which despite possessing extremely different masses, have rather standard $P$ and $\dot{P}$ for MSPs and are similar to each other. In this paper, by "progenitor NS" we denote the NS as it was born in a supernova explosion.

The binary MSPs PSR J1614-2230 and PSR J0751+1807 are both located in the galactic disk. That they do not belong to globular clusters is important for studying stellar evolution. Indeed, globular clusters are very dense systems composed of thousands of stars, where frequent stellar interactions may change the orbital parameters and sometimes even replace the companion star by another star, erasing the memory of the previous stages of evolution.

Stellar evolution theory for binary stars aims at reconstructing the different stages at the origin of the formation of PSR J1614-2230 and PSR J0751+1807 and at explaining their measured masses and other parameters, the masses of their WD companions, and the parameters of the binary orbits (collected in Table 1). In the case of PSR J1614-2230, this task was undertaken by Tauris et al. (2011) and Lin et al. (2011). While we utilize the results of Tauris et al. (2011), we concentrate on a refined description of the disk-accretion spin-up process that actually produced the observed object. The evolutionary scenario of the formation of PSR J0751+1807 will combine various elements of available evolutionary scenarios that led from a wide binary of two main sequence (MS) stars to the present $\mathrm{NS}+\mathrm{WD}$ binary. Our main results refer to the progenitor NSs

\footnotetext{
1 ATNF Pulsar Catalogue

http://www.atnf.csiro.au/people/pulsar/psrcat
}

of PSR J1614-2230 and PSR J0751+1807, which turn out to have very different masses. This may be interesting in the context of the (still incomplete) theory of the formation of NSs in core-collapse supernovæ.

The article is composed as follows. In Sect. 2, the evolutionary scenarios for the formation of PSR J1614-2230 and PSR J0751+1807 binary systems are presented. Our model for the recycling process based on Bejger et al. (2011) is summarized in Sect. 3, and the details about the EOS of dense matter, the model for the magnetic field, and its accretioninduced decay are given in Sects. 4-6, respectively. Results for PSR J1614-2230 and PSR J0751+1807 and bounds on the properties of the progenitor NS of these two pulsars are presented in Sects. 7 and 8. Section 9 contains discussions of the age of the two MSPs and of their spin frequency just after the spin-up process ended, and conclusions are given in Sect. 10. Additionally, in the Appendix we present a simple approximation of our model, which, from the currently observed properties of a given MSP, enables us to calculate the parameters of its progenitor NS. Preliminary results of this work were presented at the CompStar 2011 Workshop in Catania, Italy, May 9-12, 2011, and in Bejger et al. (2013).

\section{Evolutionary scenarios of formation of two binaries}

We begin by sketching the plausible evolutionary scenarios that could have led to the present binaries containing PSR J1614-2230 and PSR J0751+1807 with their WD companions. We assume that in both cases, the initial system is a binary of two main sequence stars of different masses: a more massive primary of initial mass $M_{1}$ will eventually produce a MSP, while a less massive secondary of initial mass $M_{2}$ will become a WD. The scenarios presented here are but brief and approximate summaries based on existing work. The main stages of the evolution leading to PSR J1614-2230 and PSR J0751+1807 binaries are schematically presented in Figs. 1 and 2. For PSR J1614-2230 we rely on Tauris et al. (2011) by selecting their Case A, with some modifications explained in the text, and reviews by de Loore \& Doom (1993) and Tauris \& van den Heuvel (2006). We use the evolutionary simulations of Lin et al. (2011) performed in the case of massive NSs born in SN explosion of the primary star, presented in Sect. 3.3 of their paper. We are aware that the evolutionary models, particularly those of massive stars, depend on many assumptions and approximations. Therefore, in what follows, we restrict ourselves to giving only approximate values of masses and timescales.

For PSR J0751+1807 binary, for which no detailed evolutionary calculations exist, we have constructed a scenario using (with some modifications) the material available in de Loore \& Doom (1993), Tauris \& van den Heuvel (2006), and Istrate et al. (2014). 
Table 2. Summary of evolutionary stages that led from the two ZAMS binaries to the present millisecond pulsar - WD binaries, for PSR J1614-2230 and PSR J0751+1807.

\begin{tabular}{ccccccccc}
\hline \hline Pulsar & & ZAMS & 1st RG & CE & SNIb/c & 2nd RG & IMXB/LMXB & Today \\
\hline PSR J1614-2230 & primary & $25 M_{\odot}$ & $5 \times 10^{6} \mathrm{yr}$ & $7 M_{\odot}$ & $1.9 M_{\odot}$ & & & $1.97 M_{\odot}$ \\
& secondary & $4.5 M_{\odot}$ & & $4.5 M_{\odot}$ & $4.5 M_{\odot}$ & $5 \times 10^{7} \mathrm{yr}$ & $5 \times 10^{7} \mathrm{yr}$ & $0.50 M_{\odot}$ \\
& $P_{\text {orb }}$ & $3 \mathrm{yr}$ & & $4 \mathrm{~d}$ & $2 \mathrm{~d}$ & & $8.7 \mathrm{~d}$ \\
\hline PSR J0751+1807 & primary & $15 M_{\odot}$ & $10^{7} \mathrm{yr}$ & $5 M_{\odot}$ & $1.2 M_{\odot}$ & & & $1.26 M_{\odot}$ \\
& secondary & $1.6 M_{\odot}$ & & $1.6 M_{\odot}$ & $1.6 M_{\odot}$ & $3 \times 10^{9} \mathrm{yr}$ & $10^{9} \mathrm{yr}$ & $0.12 M_{\odot}$ \\
& $P_{\text {orb }}$ & $5 \mathrm{yr}$ & & $1 \mathrm{~d}$ & $3 \mathrm{~d}$ & & & $6.3 \mathrm{~h}$ \\
\hline
\end{tabular}

Notes. The IMXB/LMXB column gives a rough estimate of the duration of the accretion phase onto NS at this stage. The last column contains presently measured parameters of the binaries.

A

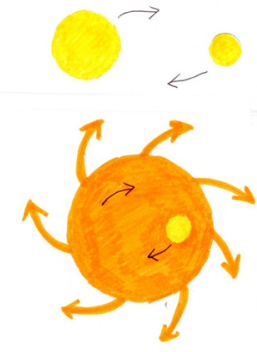

C

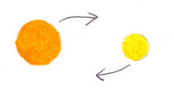

D

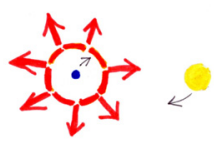

E

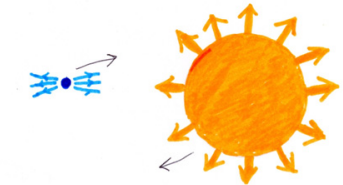

F

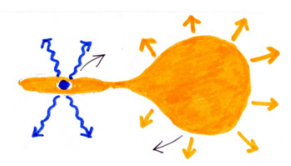

G
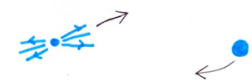

A

B

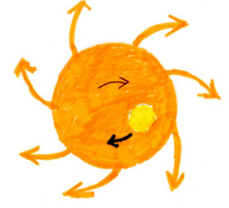

C

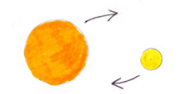

D

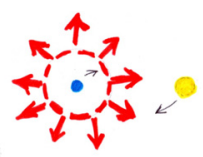

E

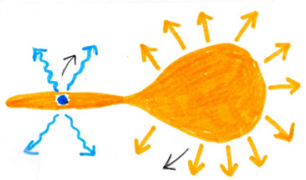

F

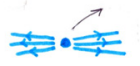

Fig. 2. Main stages of the evolution of the binary system leading to the currently observed PSR J0751+1807, accompanied by a $0.12 M_{\odot}$ WD (detailed description in Sect. 2.2). A - main sequence stage; $\mathbf{B}$ - common envelope stage (secondary inside the primary); $\mathbf{C}$ - the primary becoming a He star; $\mathbf{D}$ - core-collapse supernova explosion of the primary; $\mathbf{E}$ - Roche lobe filling of the secondary and its strong mass loss, LMXB and recycling stage; $\mathbf{F}$ - current state: compact binary MSP+WD.

Fig. 1. Main stages of the evolution of the binary system leading to currently observed PSR J1614-2230, accompanied by a $0.5 M_{\odot}$ WD (detailed description in Sect. 2.1). A - main sequence stage; $\mathbf{B}$ - common envelope stage (secondary inside the primary); $\mathbf{C}-$ the primary becoming a He star; $\mathbf{D}$ - core-collapse supernova explosion of the primary; $\mathbf{E}$ - intermediate-mass X-ray binary stage, with a very strong mass loss; F - LMXB, NS recycling stage via accretion disk, secondary mass loss via RLO; $\mathbf{G}$ - current state, wide binary MSP+WD.

It has been recently pointed out that the terminal phase of the Roche lobe overflow (RLO) stage could be associated with loss of angular momentum by the spun-up NS (Tauris 2012). This is related to the expansion of the magnetosphere accompanied by a decreasing accretion rate and the breaking of the quasi-equilibrium character of the spin evolution. The gradual switching-off of accretion occurs during the Roche lobe detachment phase (RLDP). If the duration of this phase, $t_{\text {RLDP }}$, is much shorter than the timescale for transmitting the effect of braking to the NS $t_{\text {torque }}$, then the effect of the RLDP spin-down is negligible compared to the spin-up during the LMXB stage or the intermediate-mass X-ray binary (IMXB) stage. This was shown to be the case for PSR J1614-2230 (Tauris et al. 2012). This MSP has a CO WD companion and was demonstrated to have its IMXB stage terminated by a rapid $\left(t_{\text {RLDP }} \ll t_{\text {torque }}\right)$ RLDP. However, PSR J0751+1807 has a He WD companion, and it is expected that the LMXB stage terminates there by a slow RLDP, so that the accreted mass that we calculate when neglecting the RLDP effect is an underestimate. An example of a slow RLDP is illustrated in Fig. 7 of Tauris et al. (2012), but it refers to a binary MSP that is quite different from PSR J0751+1807. It has $P=$ $5.2 \mathrm{~ms}$, instead of $3.48 \mathrm{~ms}$ for PSR J0751+1807. Incidentally, in this example the NS rotation period just before the RLDP

coincides with the present period of PSR J0751+1807 (which we get by construction at the end of our LMXB stage). The expected effect of including the RLDP braking on the accreted mass required to reproduce the present period of PSR J0751+1807 will be discussed in Sect. 10.

Predicted masses, timescales, and orbital periods referring to each stage, collected in Table 2, are but approximate estimates. We will stress the differences in the evolutionary scenarios, conditioned by the present parameters of the pulsars and their WD companions.

\subsection{PSR J1614-2230}

Binary parameters and their evolution after the primary SN explosion are taken from Case A of Tauris et al. (2011) and Sect. 3.3 of Lin et al. (2011), with some modifications for the sake of consistency between our scenario for the spin-up of the NS during the LMXB stage and the present pulsar parameters. In what follows we also use the reviews of de Loore \& Doom (1993) and Tauris \& van den Heuvel (2006). In view of the uncertainties in the evolution models, we restrict ourselves to giving only approximate values of masses and timescales.

A: main sequence $(M S)$ At the zero-age main sequence (ZAMS), $M_{1}=25 M_{\odot}$ and $M_{2}=4.5 M_{\odot}$, and the orbital period is $\sim 3$ yr. After some $5 \times 10^{6} \mathrm{yr}$ (de Loore \& Doom 1993), the primary becomes a red giant $(\mathrm{RG})$ and its envelope absorbs 
the main sequence secondary. The binary then enters the common envelope stage.

$B$ and $C$ : common envelope $(C E)$ The secondary star spirals within the primary, transferring its angular momentum to a weakly-bound envelope. As a consequence, the envelope is shed away on a timescale of $\sim 10^{3} \mathrm{yr}$. The accretion onto the secondary within the $\mathrm{CE}$ can be neglected. What remains out of the primary is a helium star with a mass $M_{\mathrm{He}, 1}=7 M_{\odot}$, in a binary with a MS secondary. For a mass $M_{\mathrm{He}, 1}>8 M_{\odot}$, the star would have collapsed into a black hole instead of a NS (Tauris \& van den Heuvel 2006). As an outcome of the CE stage, $18 M_{\odot}$ has been ejected from the binary. Frictional dissipation of kinetic energy causes the binary to shrink and shortens its orbital period to $\sim 4 \mathrm{~d}$.

D: Supernova $(S N)$ The outcome of $\mathrm{SNIb} / \mathrm{c}$ determines the initial state for the IMXB/LMXB evolution stages (Tauris et al. 2011; Lin et al. 2011). We assume $M_{\mathrm{NS}} \approx 1.9 M_{\odot}$, higher than NS masses in Tauris et al. (2011) and Lin et al. (2011) in order to be consistent with our NS spin-up scenario during the LMXB stage. As a result, in our scenario the helium star of $M_{\mathrm{He}, 1}=7 M_{\odot}$ collapses into a massive NS of $M_{\mathrm{NS}}=1.9 M_{\odot}$, while as much as $5 M_{\odot}$ is ejected in a SNIb/c explosion. The orbit becomes strongly eccentric. The angular momentum loss during the supernova explosion decreases the orbital period to $\sim 2 \mathrm{~d}$. A massive radio pulsar is born in the centre of the supernova. After a few tens of Myr of magnetic-dipole rotation braking, the pulsar period increases to a few seconds. While keeping its original surface magnetic field $\sim 10^{12} \mathrm{G}$, the NS rotates too slowly to generate radio emission and enters the pulsar graveyard. Then, $5 \times 10^{7} \mathrm{yr}$ after the ZAMS stage, the secondary leaves the MS.

$E$ and $F$ : Intermediate-mass and low-mass $X$-ray binary $\mathrm{We}$ adapt the Case A scenario of Tauris et al. (2011), which is consistent with the high NS mass scenario in Sect. 3.3 of Lin et al. 2011, with some additional comments. The binary enters the stage of intermediate-mass X-ray binary, called thus because the donor star has an initial mass of $4.5 M_{\odot}$, which is substantially higher than what is characteristic of a donor star in the initial stage of LMXBs $\left(<1 M_{\odot}\right)$. After filling its Roche lobe and starting to lose mass, the secondary becomes unstable on a thermal timescale of $\sim 10^{6} \mathrm{yr}$ (Langer et al. 2000). The mass loss via the RLO is $3.4 M_{\odot}$, so that the mass of the secondary decreases to $1.1 M_{\odot}$ (Fig. 5 of Tauris et al. 2011). The mass accreted by the NS is assumed to be negligible, at most $0.01 M_{\odot}$ (Tauris et al. 2011; Lin et al. 2011). Then the system enters the LMXB stage associated with a spin-up (recycling) of a dead pulsar via an disk accretion from its companion (donor) star. The RLO is initiated at $P_{\text {orb }}=2.2 \mathrm{~d}$, and the final period $P_{\text {orb }}=9 \mathrm{~d}$ (Tauris et al. 2011). The widening of the orbit results from the mass loss from the system, and the magnetic braking is small. Accretion onto the NS induces the dissipation of its magnetic field to its current value $\sim 10^{8} \mathrm{G}$ inferred from the measured $P$ and $\dot{P}$. During the $\sim 5 \times 10^{7} \mathrm{yr}$ of the LMXB stage, the NS is spun-up to $317 \mathrm{~Hz}$ by accreting matter from the accretion disk. The NS spin-up is not considered in Lin et al. (2011), where only NS mass and $P_{\text {orb }}$ are studied. The LMXB stage ends after the mass loss of the secondary has stopped, leaving a $0.5 M_{\odot}$ WD. The binary orbit is circularized during the LMXB stage owing to the tidal dissipation, and the orbit eccentricity goes down to $e \sim 10^{-6}$. As stressed in Tauris et al. (2011), the proposed evolutionary scenario "is only qualitative". Both Tauris et al. (2011) and Lin et al. (2011) report that the initial mass of the recycled pulsar is higher than $1.6 \pm 0.1 M_{\odot}$, while we obtain $\sim 1.9 M_{\odot}$ (see Sect. 7). As a result of the IMXB/LMXB stage, dominated by the mass loss from the system with a negligible effect of the magnetic braking, we obtain a wide binary with $P_{\text {orb }}=8.7 \mathrm{~d}$, composed of a WD of $0.5 M_{\odot}$ and a MSP of $1.97 M_{\odot}$ and $f=317 \mathrm{~Hz}$.

\subsection{PSR J0751+1807}

We assume that during the first 15 Myr, the binary evolution follows the one summarized in Fig. 16.12 of Tauris \& van den Heuvel (2006). We slightly deviate from this evolutionary track by assuming that the supernova explosion of the primary produces a $1.2 M_{\odot}$ NS. This mass is $0.1 M_{\odot}$ lower than in Tauris \& van den Heuvel (2006) and should therefore result in a slightly higher eccentricity of the post-SN binary. We also assume that, because of a weaker angular momentum loss, the orbital period after the primary SNIb/c explosion is initially $3 \mathrm{~d}$ (1 d longer than in Tauris \& van den Heuvel 2006). Together with the NS mass of $M=1.2 M_{\odot}$, this is the starting point of an evolutionary track (converging LMXB in Fig. $2 \mathrm{E}$ ) that we select from a large set calculated by Istrate et al. (2014). According to these results, magnetic braking operates at all times between the ZAMS and the final state MSP+WD. Secondly, wind mass loss from a donor star is much less than the loss via the RLO mechanism (Istrate et al. 2014). We adjust the spin-up duration and the mean accretion rate during the LMXB stage to reproduce the parameters of the present NS+WD binary. All these changes, which should be taken with a grain of salt, result from the lack of complete detailed evolutionary calculations for the currently observed binary with PSR J0751+1807.

A: Main sequence At the ZAMS $M_{1}=15 M_{\odot}$ and $M_{2}=$ 1.6 $M_{\odot}$, and the orbital period is $\sim 5 \mathrm{yr}$. In $\sim 10^{7} \mathrm{yr}$, the primary becomes a RG and absorbs the MS secondary, and a brief $\left(\sim 10^{3} \mathrm{yr}\right)$ CE stage follows.

$B$ and $C$ : Common envelope The secondary star spirals towards the centre of the primary, transferring angular momentum to the envelope of the primary. As a consequence, the envelope is shed away on a timescale of $\sim 10^{3} \mathrm{yr}$. The envelope of $10 M_{\odot}$ is ejected from the binary, kinetic energy is frictionally dissipated, the orbit shrinks, and the orbital period is reduced to $1 \mathrm{~d}$. The rest of the primary reduces to a helium star with $M_{\mathrm{He}, 1}=5 M_{\odot}$ in a binary with a MS secondary.

D: Primary supernova explosion and Roche-lobe overflow by the secondary The evolved core of the helium-star primary collapses into a light NS: $M_{\mathrm{NS}}=1.2 M_{\odot}$, with most of the mass of the primary being ejected in a SNIb/c explosion. A low-mass radio pulsar is born at the $\mathrm{SNIb} / \mathrm{c}$ centre, and the orbital period increases to $3 \mathrm{~d}$ (by construction, $1 \mathrm{~d}$ longer than in Tauris \& van den Heuvel 2006. This can be easily obtained by tuning the orbital angular momentum loss via mass loss in SNIb/c used up to this point). After a few tens of Myr, the NS enters the pulsar graveyard. Then, $3 \times 10^{9}$ yr after the ZAMS stage ${ }^{2}$, the secondary fills its Roche lobe. This is because of a rapid orbital angular momentum loss associated with a very efficient magnetic braking. The orbital period shortens by a factor of three (Istrate et al. 2014). Then the secondary overflows its Roche lobe, and the binary enters the stage of the LMXB.

E: Low-mass $X$-ray binary In the following paragraph we rely on the modelling of Istrate et al. (2014) for the evolution of the LMXB. During this phase. which lasts a few $10^{9} \mathrm{yr}$, the pulsar is spun-up by matter falling from an accretion disk; however, we estimate that periods of intense accretion, during which the essential of the spin-up takes place, occur on a time scale

2 We used $t_{\mathrm{MS}} \propto M^{-3}$ for low-mass stars as deduced from Table 11.2 of de Loore \& Doom (1993). 
of $10^{9} \mathrm{yr}$. We adopt this value in the following. The NS magnetic field is buried by the accreted matter, decreasing to a value $\sim 10^{8} \mathrm{G}$ derived from the present $\dot{P}$. The value of $P_{\text {orb }}=3 \mathrm{~d}$ at the beginning of mass transfer is below the bifurcation period $P_{\text {bif }} \sim 4$ d, in contrast to Fig. 16.12 of Tauris \& van den Heuvel (2006). The LMXB track is therefore of converging type, and $P_{\text {orb }}$ decreases in time (see Tauris \& van den Heuvel 2006 and references therein). The orbital period shortens because of an efficient angular momentum loss resulting from magnetic braking and gravitational wave radiation. In what follows we illustrate our case using an evolutionary track from a large set of tracks calculated by Istrate et al. (2014). Taking the LMXB model with initial $P_{\text {orb }}=3.2 \mathrm{~d}$ and adjusted magnetic braking, one gets a compact NS+WD binary with $P_{\text {orb }}=6.3 \mathrm{~h}$. The NS is spunup to $287 \mathrm{~Hz}$ by accretion from the disk, and the WD mass at the end is $0.16 M_{\odot}$, which is quite close to the measured mass of WD. The efficiency of accretion onto the NS is rather low (30\%), so that $70 \%$ of mass lost by the secondary leaves the binary. We assume that an appropriate small tuning of the LMXB stage can result in a decrease in the WD mass to a measured value of $0.12 M_{\odot}$. Finally, after the accretion onto the NS has stopped, the pulsar activity restarts.

$F$ : Current state The evolution of the binary at the LMXB stage is dominated by the angular momentum loss associated with an efficient magnetic braking. As a result, the present $\mathrm{MSP}+\mathrm{WD}$ binary is relativistic with an orbital period of only $6.3 \mathrm{~h}$. The orbit, which is highly eccentric after the SNIb/c explosion, has been circularized by the tidal interactions of the NS with the secondary down to $e \sim 10^{-6}$.

\subsection{Differences in evolutionary scenarios and their causes}

We have assumed that both binary systems, hereafter referred to as $\mathrm{H}$ (heavy) and L (light), resulted from the evolution of binaries that originally consisted of two ZAMS stars. Therefore, the striking differences between today's NS+WD binaries are the imprint of initial conditions. NS(H) originated in a $25 M_{\odot}$ ZAMS primary star, to be compared with a $15 M_{\odot}$ ZAMS progenitor for $\mathrm{NS}(\mathrm{L})$. Even more dramatic is the difference between the ZAMS masses of progenitors of WDs: $M_{2}(\mathrm{H})=4.5 M_{\odot} \approx 3 M_{2}(\mathrm{~L})$.

The ZAMS masses of stars in the $\mathrm{H}$ binary are significantly higher than the masses of their counterparts in the L binary. Consequently, H-binary evolution timescales are significantly shorter than L-binary ones. The formation of the first RG star in the $\mathrm{H}$ binary requires half of the time needed for this in the $\mathrm{L}$ binary. The second RG in the $\mathrm{H}$ binary is formed after $5 \times 10^{7}$ $\mathrm{yr}$, which is only $\sim 2 \%$ of the time needed for that in the L binary.

As much as $18 M_{\odot}$ is lost by the $\mathrm{H}$ binary during the $\mathrm{CE}$ stage, nearly double the mass loss by the L binary during $\mathrm{CE}$ evolution.

The H-binary orbital evolution during the IMXB-LMXB stage is dominated by the mass loss, the effect of magnetic braking being absent, and $P_{\text {orb }}$ increases from $2 \mathrm{~d}$ after SNIb/c explosion to the current value of $8.7 \mathrm{~d}$. The L binary only goes through the LMXB stage. In contrast to the $\mathrm{H}$ case, L-binary orbital evolution has to be dominated by the angular momentum loss caused by the magnetic braking, and this allows $P_{\text {orb }}$ to decrease from $3 \mathrm{~d}$ just after the $\mathrm{SNIb} / \mathrm{c}$, down to $6 \mathrm{~h}$ today, so that $P_{\text {orb }}(\mathrm{H})=35 P_{\text {orb }}(\mathrm{L})$.

\section{Spin-up by accretion}

We now briefly summarize the model for the recycling process, i.e. the spin-up of a progenitor NS by accretion of matter from a thin disk leading to the formation of a millisecond pulsar. We do not model the evolution of the binary system consisting of the NS and its companion, but only the spin-up of the NS due to accretion of matter. Our approach follows Bejger et al. (2011) and is applied here to the two pulsars PSR J1614-2230 and PSR J0751+1807. We assume that the evolution of an accreting NS can be described by a sequence of stationary rotating configurations of increasing baryon mass $M_{B}$, obtained for an assumed EOS. The increase in total stellar angular momentum $J$ is calculated by taking into account the transfer of specific orbital angular momentum of a particle accreted from a thin accretion disk. This proceeds at a distance $r_{0}$ from the centre of the NS. It results from the interaction of the disk with the NS magnetic field and is obtained using the prescription for the magnetic torque by Kluźniak \& Rappaport (2007).

The spin evolution of an accreting NS results from the interplay between the spin up due to the accretion of matter associated with angular momentum transfer and the braking due to the interaction between the NS magnetic field and the accretion disk. For details of the implementation and tests, we refer to Bejger et al. (2011). The evolution of the total angular momentum transferred to the NS of mass $M$ and radius $R$ is described by

$$
\begin{aligned}
\frac{\mathrm{d} J}{\mathrm{~d} M_{B}}=l_{\mathrm{tot}} & \equiv l\left(r_{0}\right)-l_{\mathrm{m}} \\
& =l\left(r_{0}\right)-\frac{\mu^{2}}{9 r_{0}^{3} \dot{M}}\left(3-2 \sqrt{\frac{r_{\mathrm{c}}^{3}}{r_{0}^{3}}}\right),
\end{aligned}
$$

with the magnetic moment $\mu=B R^{3}$. Here, $l\left(r_{0}\right)$ is the specific angular momentum of an infalling particle at the inner boundary of the disk $r_{0}$ and $l_{\mathrm{m}}$ (magnetic torque divided by the accretion rate $^{3}$ ) describes the interaction of the NS magnetic field with the accretion disk. One can define three characteristic lengths of the problem: the magnetospheric radius $r_{\mathrm{m}}=(G M)^{-1 / 7} \dot{M}^{-2 / 7} \mu^{4 / 7}$, the corotation radius $r_{\mathrm{c}}=\left[G M /\left(4 \pi^{2} f^{2}\right)\right]^{1 / 3}$, and the location of the relativistic marginally stable orbit $r_{\mathrm{ms}}$. Then, the inner boundary $r_{0}$ is determined by the condition of vanishing viscous torque leading to an algebraic equation containing these lengths:

$\frac{1}{2} f_{\mathrm{ms}}\left(r_{0}\right)=\left(\frac{r_{\mathrm{m}}}{r_{0}}\right)^{7 / 2}\left(\sqrt{\frac{r_{\mathrm{c}}^{3}}{r_{0}^{3}}}-1\right)$ with $f_{\mathrm{ms}}(r)=\frac{2}{\Omega r} \frac{\mathrm{d} l}{\mathrm{~d} r}$,

which is a dimensionless function describing the location of the marginally stable orbit for rotating NSs in General Relativity, defined by $f_{\mathrm{ms}}\left(r_{\mathrm{ms}}\right)=0$.

The modeling of the accretion phase consists in adjusting the value of the mass $M_{0}$ (or equivalently the baryonic mass $M_{\mathrm{B} 0}$ ), the magnetic field $B_{\mathrm{i}}$ of the progenitor NS, and the mean accretion rate $\dot{M}$ (accretion rate averaged over the whole accretion process) so that at the end of the accretion process, the NS has its parameters, i.e. its mass $M$ (baryonic mass $M_{B}$ ) and magnetic field $B$, coinciding with those of a given millisecond pulsar. We also consider that the post-accretion frequency is equal to its currently observed value $f$. The validity of this assumption is discussed in Sect. 9. For a given final configuration (i.e. $M, P$, and $B$ ), a family of sets of three parameters: $M_{0}$ (hence the amount of accreted matter $M_{\mathrm{acc}}=M_{B}-M_{\mathrm{B} 0}$ ), $B_{\mathrm{i}}$ and $\dot{M}$ (or equivalently the duration of the accretion phase $\left.\tau_{\mathrm{acc}}=M_{\mathrm{acc}} / \dot{M}\right)$, is obtained. As a consequence, a choice of $\tau_{\text {acc }}$ imposes $M_{\text {acc }}$ and $B_{\mathrm{i}}$.

For simplicity we adopt the notation $\dot{M}$ for the accretion rate instead of $\dot{M}_{B}$. The accretion rate is indeed described in terms of baryon mass $M_{B}$, since this number is well defined for the binary system, see Bejger et al. (2011). 


\section{Equations of state}

The EOS of the dense cores of NSs is still poorly known. This is due to, on the one hand, a lack of knowledge of strong interaction in dense matter and, on the other hand, deficiencies in the available many-body theories of dense matter. This uncertainty is reflected in a rather broad scatter of theoretically derived and EOSdependent maximum allowable mass for NSs (see e.g. Haensel et al. 2007). Fortunately, the recent measurements of the mass of PSR J1614-2230 as $M=1.97 \pm 0.04 M_{\odot}$ (Demorest et al. 2010) and PSR J0348+0432 as $M=2.01 \pm 0.04 M_{\odot}$ (Antoniadis et al. 2013 ) introduce a fairly strong constraint on $M_{\max }$. It implies that the (true) EOS is rather stiff. To illustrate the remaining uncertainty, we considered two different models for dense matter:

DH (Douchin \& Haensel 2001). It is a non-relativistic model for the simplest possible composition of matter: neutrons, protons, electrons, and muons in $\beta$ equilibrium. The energy density functional is based on the SLy4 effective nuclear interaction. The model describes both the dense liquid core of the NS and its crust in a unified way. This EOS yields a maximum mass $M_{\max }=2.05 M_{\odot}$ and a circumferential radius at maximum mass $R_{M_{\max }}=10.0 \mathrm{~km}$ (for a non-rotating configuration).

BM (Bednarek et al. 2012). It is a nonlinear relativistic model that allows for a softening owing to the appearance of hyperons at a density of $\sim 6 \times 10^{14} \mathrm{~g} \mathrm{~cm}^{-3}$. The nonlinear Lagrangian includes up to quartic terms in the meson fields. The meson fields $\sigma, \omega$, and $\rho$ are coupled to nucleons and hyperons, and hidden-strangeness meson fields $\sigma^{\star}$ and $\phi$ only couple to hyperons. The vector meson $\phi$ generates highdensity repulsion between hyperons. The EOS is calculated in the mean field approximation. It yields $M_{\max }=2.03 M_{\odot}$ and $R_{M_{\max }}=10.7 \mathrm{~km}$ (for a non-rotating configuration).

Our models for baryonic matter do not include the $\Delta$ resonance (first excited state of the nucleon) as a real constituent of dense NS matter. In the vacuum, the $\Delta$ is very different from hyperons. The instability of hyperons is due to the weak interactions, with a typical half width to the rest-energy ratio for the lightest hyperon $\Lambda: \Gamma_{\Lambda} / m_{\Lambda} c^{2} \approx 10^{-14}$. Hyperons in the vacuum are stable with respect to the strong interaction processes. In contrast, the $\Delta$ is unstable because of the strong interactions, and $\Gamma_{\Delta} / m_{\Delta} c^{2} \approx 0.1$. In dense matter, the rest energy of a baryon should be replaced by the self-energy (in-medium mass), when taking the interactions with other particles into account, as well as the exclusion principle blocking some processes that were allowed in the vacuum. For example, when the $\Delta$ is stabilized in dense matter, this means a decrease in $\Gamma_{\Delta}$ from $120 \mathrm{MeV}$ in the vacuum to zero in dense matter. It has been argued that these medium-induced effects increase the threshold density for the appearance of stable $\Delta$ in dense matter above the maximum density reachable in NSs (e.g. Sawyer 1972; Nandy 1974; Glendenning 1985). In the relativistic mean field approximation, the threshold density for the $\Delta$ depends strongly on the in-medium $\Delta$ mass and the completely unknown $\Delta$ coupling constant to the isovector-vector meson $\rho$. Recently, the question of a possible presence of $\Delta$ in NSs was revived in Drago et al. (2014a,b). Viewing the uncertainties in the in-medium effects on the $\Delta$ at supra-nuclear densities, however, we do not consider them in NS cores.

\section{Magnetic field of a pulsar}

Only an estimate of the magnetic field $B$ of a given pulsar can be obtained if its rotational period $P$ and period derivative $\dot{P}$ are known. Assuming that the pulsar is a rotating magnetic dipole and that its loss of rotational energy originates in the emission of magneto-dipole radiation alone, one derives the following classical dipole formula (see e.g. Haensel et al. 2007):

$B=\sqrt{\frac{3 c^{3} I P \dot{P}}{8 \pi^{2} R^{6}} \frac{1}{\sin ^{2} \alpha}}$,

where $\alpha$ is the angle between the rotation and magnetic axes: $0<\alpha \leqslant 90^{\circ}$. This formula describes a spinning dipole in a vacuum, meaning a dipole without plasma in the magnetosphere. It breaks down for the case of aligned rotator, $\alpha=0^{\circ}$. However, a more physically sound formula has been derived by Spitkovsky (2006), approximating solutions of the force-free relativistic MHD equations in the magnetosphere filled with plasma for both aligned and oblique rotators. For $0 \leqslant \alpha \leqslant 90^{\circ}$,

$B=\sqrt{\frac{c^{3} I P \dot{P}}{4 \pi^{2} R^{6}} \frac{1}{1+\sin ^{2} \alpha}}$.

To test the dependence of the modelling on the estimate of the magnetic field of the pulsar during the recycling process, two different models are considered in the following, each of them corresponding to a given value of the parameter $\beta$ in the equation

$B=\beta \sqrt{P_{\mathrm{ms}} \dot{P}_{-20}} \frac{\sqrt{I_{45}}}{R_{6}^{3}} 10^{8} \mathrm{G}$.

In the above formula, $P_{\mathrm{ms}}$ is the period in $\mathrm{ms}, \dot{P}_{-20}$ the period derivative in units of $10^{-20} \mathrm{~s} \mathrm{~s}^{-1}, I_{45}$ the moment of inertia in units of $10^{45} \mathrm{~g} \mathrm{~cm}^{2}$, and $R_{6}$ the radius in $10^{6} \mathrm{~cm}$. The dimensionless parameter $\beta$ takes the following value:

Model $(a): \beta=1.01$. This corresponds to taking the lowest possible value of the magnetic field for the magnetic dipole obtained from the dipole formula (3) for an orthogonal rotator $\left(\alpha=90^{\circ}\right)$;

Model $(b): \beta=0.83$ derived using Spitkovsky's formula (4) for an aligned rotator $\left(\alpha=0^{\circ}\right)$.

The second model is consistent with the model of an accretion disk presented in Kluźniak \& Rappaport (2007), as used in our approach that assumes that the magnetic field and the rotation axes are aligned. The first model, which is widely used in the literature, serves as a reference for comparison with previous works (e.g. Bejger et al. 2011).

With the above formula, one can calculate the value of the magnetic field of the pulsar at the end of the spin-up process consistently with the EOS, by taking the values of $I$ and $R$ for a NS rotating at the frequency and mass of interest. In the last column of Table 1 , values $B_{0}$ of the canonical derived magnetic field are quoted, i.e., values obtained from Eq. (5) for the magnetic field model (a) and considering a NS with canonical values for the radius and the moment of inertia: $R_{0}=10 \mathrm{~km}$ and $I_{0}=10^{45} \mathrm{~g} \mathrm{~cm}^{2}$.

\section{Decay of NS magnetic field in LMXBs and IMXBs}

Although observations do not give any evidence of magnetic field decay during the radio pulsar phase, a substantial magnetic field decay (by some four orders of magnitude) is expected to occur during the "recycling" process in a LMXB, leading to the 
Table 3. PSR J1614-2230: values for a NS rotating at $f=317 \mathrm{~Hz}$ of the moment of inertia $I_{45}$ (in units of $10^{45} \mathrm{~g} \mathrm{~cm}^{2}$ ), the equatorial radius $R_{6}$ (in $10^{6} \mathrm{~cm}$ ), and of the magnetic field $B_{8}\left(\right.$ in $10^{8} \mathrm{G}$ ).

\begin{tabular}{cccccc}
\hline \hline EOS & Mass $\left(M_{\odot}\right)$ & $B$ model & $I_{45}$ & $R_{6}$ & $B_{8}$ \\
\hline DH & 1.97 & (a) & 1.99 & 1.08 & 1.98 \\
DH & 1.97 & (b) & 1.99 & 1.08 & 1.62 \\
BM & 1.97 & (a) & 2.19 & 1.18 & 1.57 \\
BM & 1.93 & (a) & 2.22 & 1.21 & 1.48 \\
BM & 2.01 & (a) & 2.10 & 1.13 & 1.77 \\
\hline
\end{tabular}

Notes. The last is obtained from Eq. (5) for the different models of magnetic field, the two EOSs, and the extrema and central values of the measured mass interval at $1 \sigma$ level.

formation of a millisecond pulsar (Taam \& van den Heuvel 1986, for a review see Colpi et al. 2001).

The theoretical modelling of the accretion-induced decay of $B$ is a challenging task (Zhang \& Kojima 2006, for a review see Bejger et al. 2011). Considering current uncertainties in the modeling of the magnetic field decay accompanying the spin-up phase of a given millisecond pulsar, we employ phenomenological models, based to some extent on observations of NSs in LMXBs. Taam \& van den Heuvel (1986) analysed several LMXBs of different ages. They suggested a possible inverse correlation between $B$ and the total amount of accreted material. This suggestion was later confirmed in a study by van den Heuvel \& Bitzaraki (1995). After analysing a subset of LMXBs, Shibazaki et al. (1989) proposed to approximate the inverse correlation between $B$ and accreted mass by a formula:

$B\left(M_{\mathrm{acc}}\right)=B_{\mathrm{i}} /\left(1+M_{\mathrm{acc}} / m_{B}\right)$

The scaling constant $m_{B}$ controls the pace of dissipation of $B$ with increasing $M_{\text {acc }}$. The values $m_{B}=10^{-4}, 10^{-5} M_{\odot}$ are both consistent with the observed or estimated $P, B$, and $M_{\text {acc }}$ of binary and isolated millisecond radio pulsars (Shibazaki et al. 1989; Francischelli et al. 2002). In the following, we adopt the value $m_{B}=10^{-5} M_{\odot}$ unless stated otherwise. The limitations and uncertainties of Eq. (6) have been reviewed in detail in Bejger et al. (2011). We use Eq. (6) as our baseline description for magnetic field decay in LMXBs and IMXBs. However, we also tested other phenomenological models for the magnetic field decay, such as the ones introduced in Kiel et al. (2008), Osłowski et al. (2011), and Wijers (1997). Our results depend weakly on the choice of a specific model (for details see Fortin 2012).

The aim of our analysis is to obtain evolutionary tracks that lead to the formation of a millisecond pulsar whose mass, rotational frequency, and magnetic field are equal to the currently observed values of PSR J1614-2230 or PSR J0751+1807 in order to infer some properties of the progenitor NS of these two extreme millisecond pulsars.

\section{PSR J1614-2230: lower bounds on $\dot{M}$ and the progenitor NS mass}

Five different setups are employed in modelling the accretion phase leading to the PSR J1614-2230 configuration: for the DH EOS, the two models for the magnetic field are used, and for the BM EOS, three values of the mass of the millisecond pulsar corresponding to the lower, upper, and central values of the $1 \sigma$ mass interval are taken into account. Their properties are indicated in Table 3.

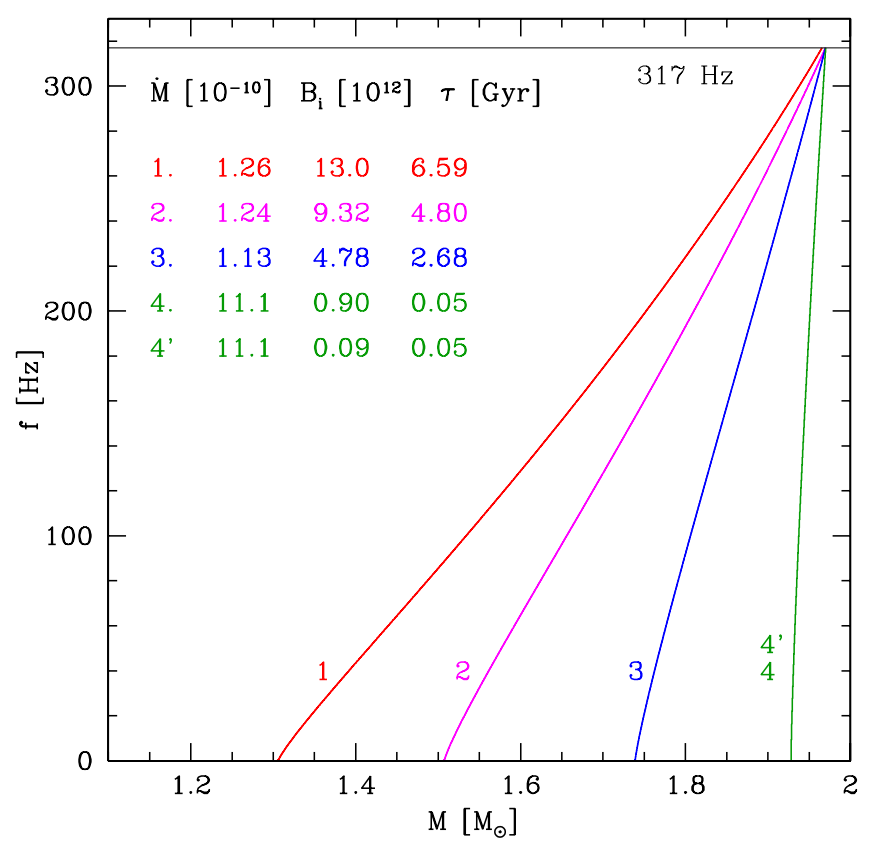

Fig. 3. Example of spin-up tracks of accreting NSs leading to the final configuration of PSR J1614-2230 for the BM EOS and for model (a) for the magnetic field decay. For each track, the mean accretion rate (in $M_{\odot} / \mathrm{yr}$ ), the initial magnetic field (in $\mathrm{G}$ ), and the duration of the accretion phase are indicated.

In Fig. 3 different spin-up tracks, i.e. the change in the spin frequency with the mass of the accreting NS, are shown for the BM EOS, the model (a) for the magnetic field, and for a set of initial (pre-accretion) masses, magnetic fields of the progenitor NS, and accretion rates. At the end of the recycling process, the final $M, B$, and $f$ match the present-day parameters of PSR J1614-2230.

As noted in Bejger et al. (2011), spin-up tracks with a nonzero initial frequency, which is expected for newly born NSs, are indistinguishable from those with a zero initial frequency on a time scale of $10^{6}$ years, which is much shorter than the ones typical of the recycling process. Spin-up tracks 1, 2, and 3 in Fig. 3 are only plotted for illustrative purposes since the duration of the accretion phase needed to reach PSR J1614-2230 configuration is too long to be physically relevant in accordance with Sect. 2.

Tracks 4 and $4^{\prime}$, which are indistinguishable from one another, are obtained for $m_{B}=10^{-5}$ and $10^{-4} M_{\odot}$, respectively. For a given set of final magnetic field, mass, and frequency, models with $m_{B}=10^{-4}$ and $10^{-5} M_{\odot}$ give equal values for the initial mass and the accretion rate. As a consequence of Eq. (6), initial magnetic fields are one order of magnitude larger for tracks with $m_{B}=10^{-5} M_{\odot}$ than for the ones with $m_{B}=10^{-4} M_{\odot}$. Track 4, calculated for $m_{\mathrm{B}}=10^{-5} M_{\odot}$, is obtained for an initial magnetic field $B_{\mathrm{i}} \sim 10^{12} \mathrm{G}$, consistent with the inferred magnetic field of isolated radio pulsars $B \sim 10^{11}-10^{13} \mathrm{G}$ (Manchester et al. 2005). For the value $m_{B}=10^{-4} M_{\odot}$ used in Bejger et al. $(2011,2013)$ and shown by track $4^{\prime}, B_{\mathrm{i}} \sim 10^{11} \mathrm{G}$.

In the following two quantities are used: the amount of accreted matter given by the relation $M_{\mathrm{acc}}=M_{B}-M_{\mathrm{B} 0}$ and the duration of the accretion phase: $\tau_{\text {acc }}=M_{\text {acc }} / \dot{M}$. To reach the mass of PSR J1614-2230, a low-mass progenitor NS has to accrete more matter than a high-mass one so it undergoes a stronger decay of its magnetic field according to Eq. (6). Consequently, its pre-accretion magnetic field had to be larger. The duration of the 


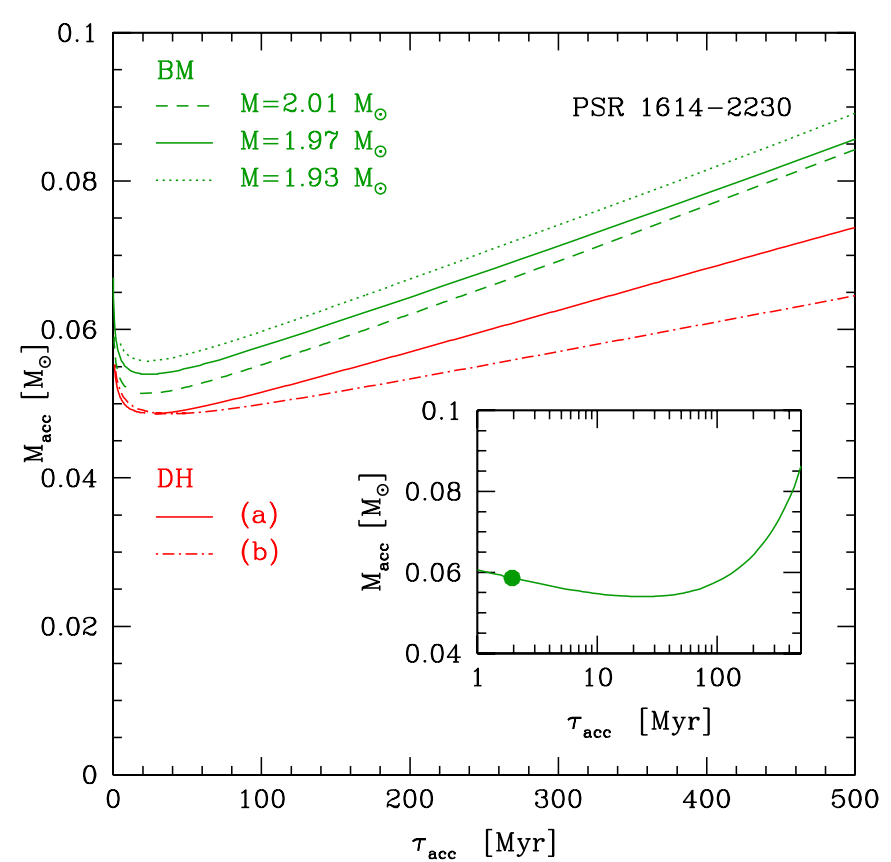

Fig. 4. Mass of accreted matter as a function of the time needed to spinup the progenitor NS to the observed properties of PSR J1614-2230 (i.e., $M, B$, and $f$ ) for the five models indicated in Table 3 . The dot corresponds to a spin-up track proceeding at a rate equal to the Eddington accretion rate $\dot{M}_{\text {Edd }} \sim 3 \times 10^{-8} M_{\odot} /$ yr; the accretion timescale is then $\sim 2$ Myr. For tracks with $\tau_{\text {acc }} \lesssim 2 \mathrm{Myr}, \dot{M}$ is greater than $\dot{M}_{\text {Edd }}$.

accretion phase is also longer for a low-mass progenitor than a high-mass one. Figures 4 and 5 show the relation between the accretion time $\tau_{\text {acc }}$ and the amount of accreted matter $M_{\text {acc }}$ and the mean accretion rate needed to reach PSR J1614-2230 in its current configuration. Both figures show weak dependence on the final NS mass and on the model of magnetic field (i.e. magnetic dipole or Spitkovsky's model). Results for $m_{B}=10^{-4}$ and $10^{-5} M_{\odot}$ are indistinguishable.

The minimum amount of accreted mass necessary for a NS to become a millisecond pulsar is reached for a finite value of $\tau_{\text {acc }}$, which corresponds to a minimum of the function $M_{\text {acc }}\left(\tau_{\text {acc }}\right)$ (see Figs. 4 and 7). For a final configuration corresponding to PSR J1614-2230, the minimum is obtained for $\dot{M}^{\text {min }} \sim 2 \times$ $10^{-9} M_{\odot} /$ yr and $\tau_{\text {acc }}^{\min } \sim 20-25 \mathrm{Myr}$. The existence of this minimum is a consequence of the fact that for accretion rates higher than $\dot{M}^{\text {min }}$, the magnetic torque, or equivalently $l_{\mathrm{m}}$ in Eq. (1), changes its sign, becoming positive (see Appendix A). The value of $\tau_{\mathrm{acc}}^{\mathrm{min}}$ is significantly less than the timescales presented in Figs. 3 and 6, and along all the tracks discussed in the following, $l_{\mathrm{m}}$ is always positive and thus counteracts $l\left(r_{0}\right)$. For an unrealistically short duration of the recycling $\tau_{\text {acc }} \lesssim 2 \mathrm{Myr}$, the model predicts that accretion proceeds at a higher rate than the Eddington rate $\dot{M}_{\text {Edd }} \sim 3 \times 10^{-8} M_{\odot} /$ yr (Tauris et al. 2012), as indicated in Fig. 4.

Model (b) gives lower values for $B$ than dipole model (a) at all stages of the recycling process. Therefore, the magnetic torque that opposes the spin up of the accreting NS is less for model (b), making the recycling process more efficient. Thus less accreted matter is needed to reach the current pulsar parameters. Moreover, since $M_{\text {acc }}$ is lower for model (b), so is the mean accretion rate in the recycling process for given accretion time $\tau_{\text {acc }}$ (see Fig. 5).

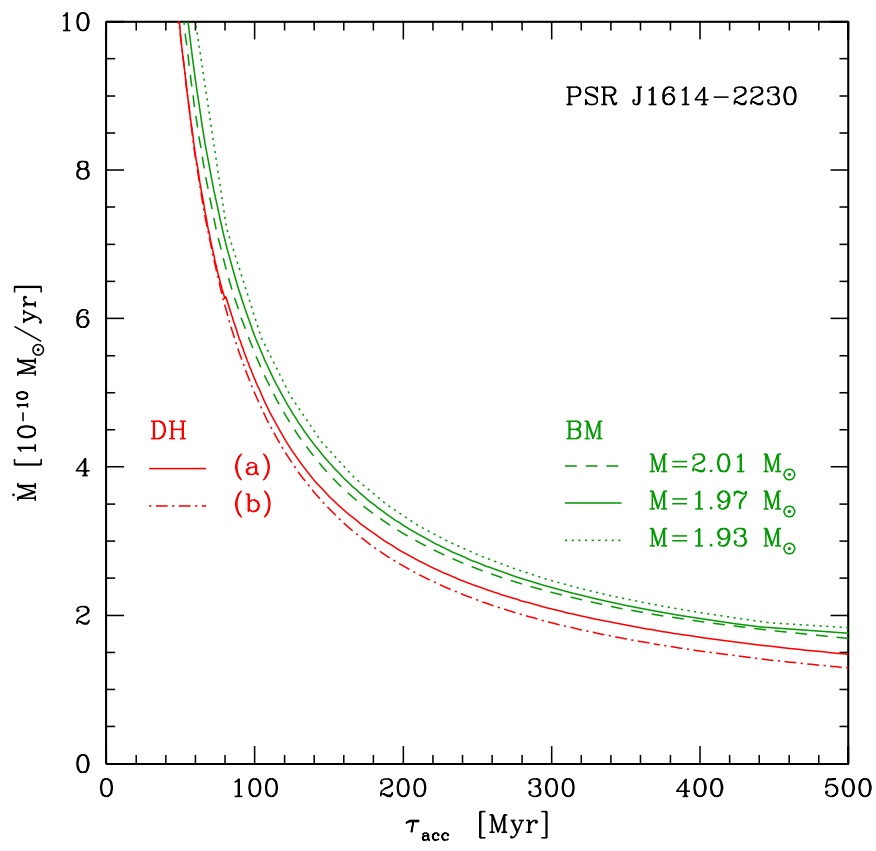

Fig. 5. Mean accretion rate versus duration of the accretion phase for DH and BM EOS needed to reach PSR J1614-2230 configuration.

Using evolutionary arguments one can constrain the minimal birth mass $M_{0}$, at the onset of accretion. For an accretion phase lasting at most $\simeq 50 \mathrm{Myr}$ (see Sect. 2 and Tauris et al. 2011), the progenitor NS must have accreted less than $\simeq 0.06 M_{\odot}$. Therefore the progenitor NS was born as massive independently of the EOS: $M_{0} \simeq 1.9 M_{\odot}$. Such a configuration is illustrated by the spin-up track 4 in Fig. 3. The value $M_{\text {acc }} \simeq 0.06 M_{\odot}$ should be considered as a lower limit, since we do not model the evolution of the binary system as in Tauris et al. (2011) or take the possible ejection of matter from the magnetosphere or instabilities in the accretion disk into account. Moreover, in our model the spin-up is assumed to be maximally efficient i.e., all angular momentum from the accreted matter is transferred to the NS. If the efficiency of the accretion process is reduced by 50 per cent (see discussion in Bejger et al. 2011), then our calculations show that the accretion of $M_{\mathrm{acc}} \simeq 0.11 M_{\odot}$ is necessary to spin up the NS to PSR J1614-2230 current configuration in 50 Myr. The same amount of accreted matter is required if accretion does not proceed from $r_{0}$ but from the magnetospheric radius $r_{\mathrm{m}}$ and with no magnetic torque, unlike in Eq. (1). Finally, as shown in Bejger et al. (2011), if accretion proceeds from the marginally stable orbit $r_{\mathrm{ms}}, M_{\mathrm{acc}}=0.076 M_{\odot}$. Therefore the mass $M_{0} \simeq 1.9 M_{\odot}$ of the NS at birth is an upper limit.

Assuming that the accretion time is well-constrained, one can also estimate the mean accretion rate using the currently observed parameters of PSR J1614-2230. Figure 5 gives $\dot{M}>$ (1.0-1.4) $\times 10^{-9} M_{\odot} / \mathrm{yr}$, the lower value corresponding to the DH EOS.

Modelling the evolution and dynamics of the binary system, Tauris et al. (2011) calculated that the NS accreted $\sim 0.3 M_{\odot}$ during $50 \mathrm{Myr}$, i.e. an averaged accretion rate of $\dot{M} \simeq 6 \times$ $10^{-9} M_{\odot} / y r$. The discrepancy between these results and ours stems from the fact that we do not model the evolution of the binary and of the donor star and, to a lesser extent, from a different model for the spin-up phase. 
Table 4. PSR J0751+1807: analogue of Table 3.

\begin{tabular}{cccccc}
\hline \hline EOS & Mass $\left(M_{\odot}\right)$ & $B$ model & $I_{45}$ & $R_{6}$ & $B_{8}$ \\
\hline DH & 1.26 & (a) & 1.19 & 1.19 & 1.09 \\
DH & 1.26 & (b) & 1.19 & 1.19 & 0.88 \\
BM & 1.26 & (a) & 1.51 & 1.36 & 0.80 \\
BM & 1.12 & (a) & 1.29 & 1.37 & 0.73 \\
BM & 1.40 & (a) & 1.74 & 1.36 & 0.87 \\
\hline
\end{tabular}

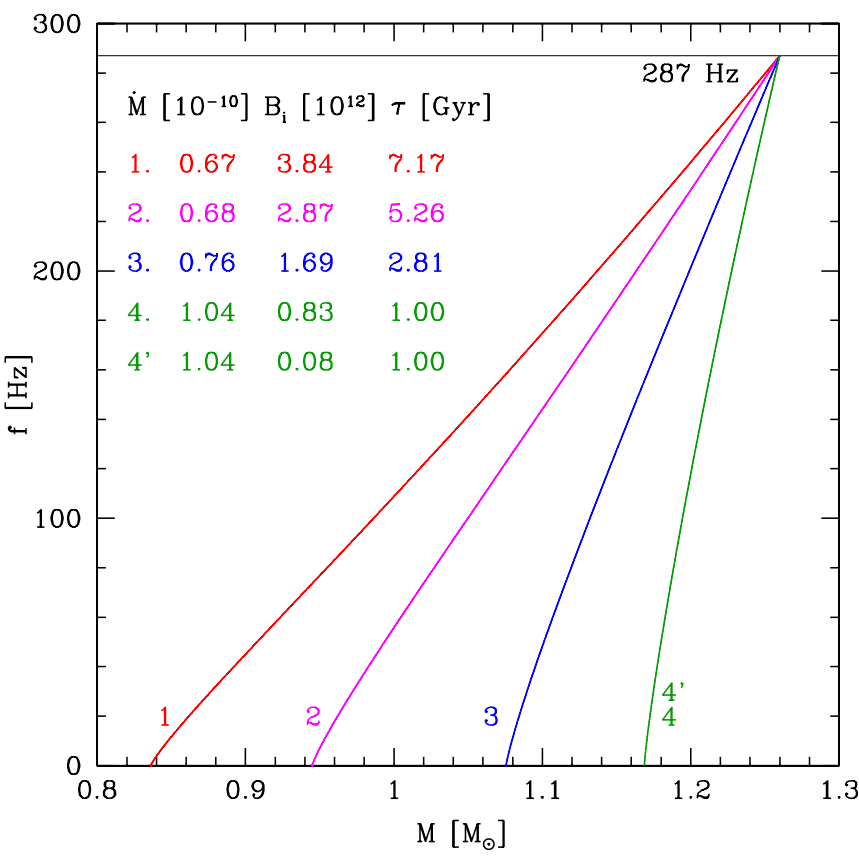

Fig. 6. Similar to Fig. 3, but for the pulsar J0751+1807.

\section{PSR J0751+1807: lower bounds on $\dot{M}$ and $M_{0}$}

A similar approach was used for PSR J0751+1807. Properties of the five models are presented in Table 4, and results are shown in Figs. 6-8. As before, spin-up tracks 1, 2, and 3 in Fig. 6 are plotted for illustrative purposes, and tracks 4 and $4^{\prime}$ are obtained for $m_{B}=10^{-5}, 10^{-4} M_{\odot}$ respectively. The figures are remarkably similar to the ones obtained for PSR J1614-2230. As for PSR J1614-2230, the only difference between results for $m_{B}=10^{-4}$ and $10^{-5} M_{\odot}$ lies in the value of the initial magnetic field.

For PSR J1614-2230, however, the amount of accreted matter needed to spin up the pulsar and the mean accretion rate decreases with increasing final mass of the pulsar. This is visualized by the order of the curves corresponding to masses 1.93, 1.97, and 2.01 $M_{\odot}$ in Figs. 4 and 5. This behaviour is opposite to what is found for PSR J0751+1807 (Figs. 7 and 8). The reason is the non-monotonic dependence of the moment of inertia $I$ (and total angular momentum of a star rotating at a fixed frequency) on the stellar mass $M$ (Bejger 2013). For the BM EOS, for example, for masses lower than $1.8 M_{\odot}, I$ increases with $M$ and a higher mass corresponds to a larger total angular momentum. As a consequence, for an equal transfer of angular momentum by accretion, a longer time is needed to spin up the star to a given frequency. The situation is opposite close to the maximum mass (for $M>1.85 M_{\odot}$ for BM model) where $I$ decreases with $M$ (see Tables 3 and 4).

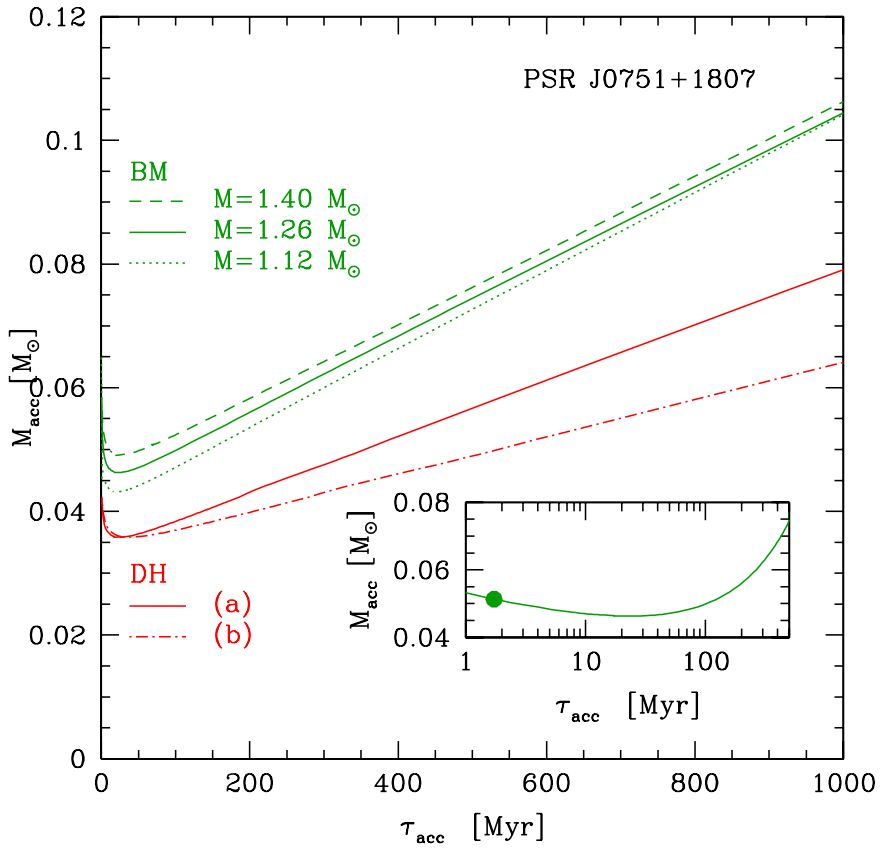

Fig. 7. Similar to Fig. 4, but for the pulsar J0751+1807.

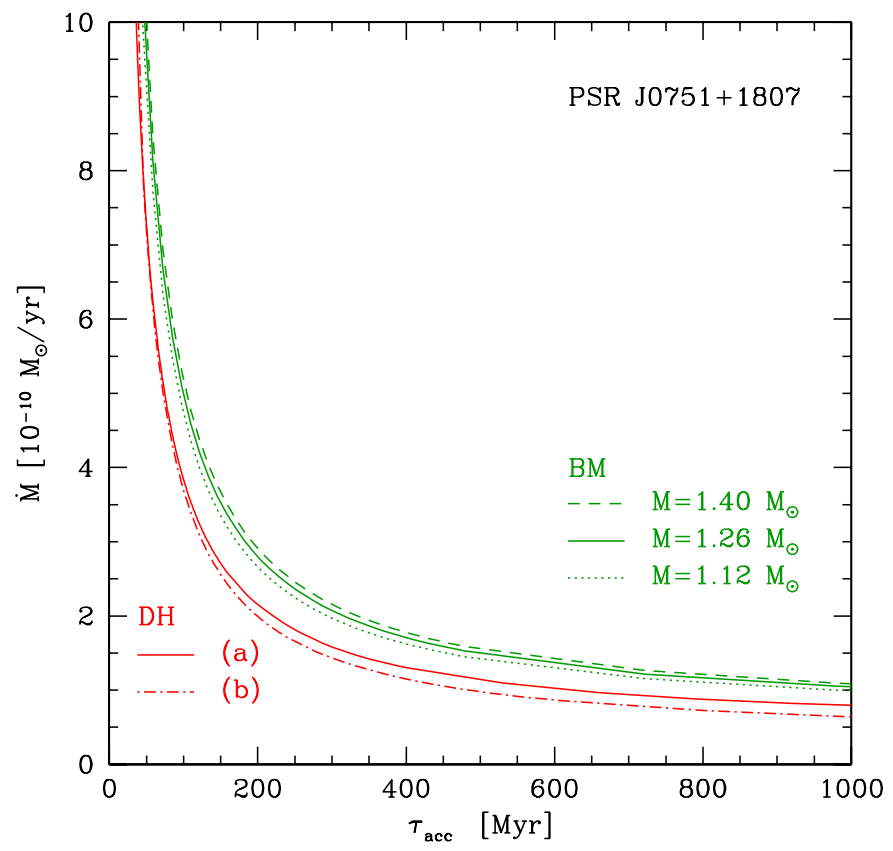

Fig. 8. Similar to Fig. 5, but for the pulsar J0751+1807.

As Fig. 7 indicates, assuming that the accretion proceeds at a rate lower than the Eddington rate for 1 Gyr (see Sect. 2), the progenitor NS of PSR J0751+1807 acquired $\sim 0.06-0.10 M_{\odot}$, which implies that it was born with a very low mass, $\sim 1.05-1.30 M_{\odot}$.

The wide range of possible birth masses for PSR J1614-2230 and PSR J0751+1807 puts stringent constraints on the modelling of supernova explosion and their outcome and on the EOS of hot dense matter, which is relevant for newly born NSs. 


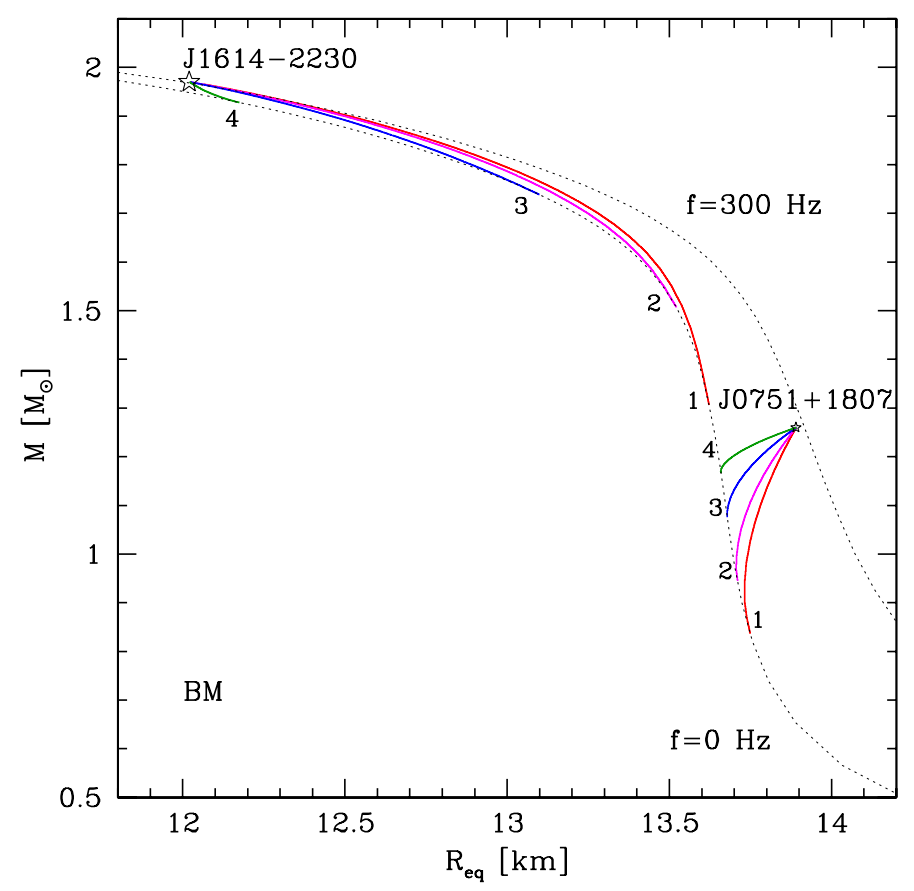

Fig. 9. Mass $M$ vs. equatorial radius $R_{\text {eq }}$ diagram and spin-up tracks of the two pulsars. The colour code and the numbers labelling the tracks correspond to the ones used in Figs. 3 and 6. For comparison relations between the mass and the equatorial radius of equilibrium configurations are shown for $f=0$ and $300 \mathrm{~Hz}$. The large open-star symbol corresponds to the PSR J1614-2230 configuration and the small one to PSR J0751+1807.

Figure 9 shows the spin-up tracks in the mass $M$ vs. equatorial radius $R_{\mathrm{eq}}$ diagram for the two pulsars. During the recycling phase, the two NSs undergo a remarkably different evolution in the $M-R_{\text {eq }}$ diagram: the equatorial radius of PSR $\mathrm{J} 0751+1807$ increases, while the one of PSR J1614-2230 substantially decreases. The role of rotation at frequency $\sim 300 \mathrm{~Hz}$ is much more important for the structure and oblateness of a star with the mass $1.3 M_{\odot}$ and radius $14 \mathrm{~km}$ than for a much more compact NS with $M$ close to the maximum mass.

\section{The age of the MSPs and their true initial spin period}

The present spin period of MSPs is expected to be longer than the period at the end of the spin-up phase. The pulsars' spindown age can be estimated with the formula $\tau_{\mathrm{PSR}}=P / 2 \dot{P}$. For the values given in Table 1 , one obtains $\tau_{\mathrm{PSR}}^{\mathrm{H}} \stackrel{\tau_{\mathrm{PSR}}}{=} 5.2 \mathrm{Gyr}$ and $\tau_{\text {PSR }}^{\mathrm{L}}=7.7$ Gyr. These values should be treated as upper limits on $\tau_{\text {PSR }}$ since the measured $\dot{P}$ is larger than the true $\dot{P}$ because of the transverse motion of the binary system (Shklovskii 1970). This effect turns out to be negligible for the low-mass pulsar (about $\sim 1 \%$ ). It may be crucial for the high-mass pulsar since the true $\dot{P}$ could be more than one order of magnitude smaller than the observed one (Bhalearo \& Kulkarni 2011). However this result is very sensitive to the uncertainties in the determination of the distance and proper motion of PSR J1614-2230. Moreover, the spin-down age has been shown not to provide a reliable age estimate, in particular for MSPs, see e.g. Kiziltan \& Thorsett (2010).

The loss of the tenuous WD envelope marks the end of the LMXB stage, which leads to the formation of a WD+MSP binary. In both cases, L-binary with PSR J0751+1807 and H-binary with PSR J1614-2230, the heating of the WD surface by the MSP irradiation can be neglected. This is quite natural for the $\mathrm{H}$ binary with $P_{\text {orb }}=8.7 \mathrm{~d}$. It is far from being obvious for the L-binary with $P_{\text {orb }}=6.3 \mathrm{~h}$, but Bassa et al. (2006) found "a surprising lack of evidence for any heating". Therefore, the age of the MSPs in both the $\mathrm{L}$ and $\mathrm{H}$ binaries can be obtained from modelling the cooling of their WD.

Using their own observations of WD(L) Bassa et al. (2006) constrained its effective temperature $T_{\text {eff }}$, radius, and the composition of its atmosphere. They found $T_{\mathrm{eff}} \approx 4000 \mathrm{~K}$ for the most likely pure $\mathrm{He}$ (or strongly He-dominated) atmosphere. Such a composition of the atmosphere is a puzzle (see discussion in Bassa et al. 2006). We applied a cooling curve obtained for a $0.15 M_{\odot}$ He-core WD with a He envelope calculated by Hansen \& Phinney (1998) and obtained $\tau_{\mathrm{wD}}^{\mathrm{L}} \approx 2.4 \mathrm{Gyr}$. We assume that this is the age of PSR J0751+1807.

The WD companion of PSR J1614-2230 was discovered in the optical observations by Bhalearo \& Kulkarni (2011) and in the following, we summarize their results. The optical colours, combined with a mass of $0.5 M_{\odot}$, indicate that the $\mathrm{WD}(\mathrm{H})$ has a $\mathrm{C} / \mathrm{O}$ core and an $\mathrm{H}$ atmosphere. Cooling sequences calculated by Chabrier et al. (2000) predict that such a WD reaches the inferred absolute magnitude in the $R$-band $M_{R} \approx 13.7$ obtained in Bhalearo \& Kulkarni (2011) after $\tau_{\mathrm{wD}}^{\mathrm{H}} \approx 2.2$ Gyr. This is the age we assume for PSR J1614-2230.

Using the measured $\dot{P}$ and assuming a braking index $n=3$, which is consistent with the observed population of MSPs (Kiziltan \& Thorsett 2010), the initial period of a pulsar at the beginning of the slowing down phase can be estimated: $P_{\text {init }}=$ $P_{\text {obs }}\left[1-(n-1) \tau_{\text {wD }} \dot{P} / P\right]^{1 /(n-1)}$, where $P_{\text {obs }}$ is the currently observed period. Here, $P_{\text {init }}$ is shorter than $P_{\text {obs }}$ by about $20 \%$ for both pulsars. For the light pulsar, the amount of accreted matter necessary to spin it up not to $P_{\text {obs }}$ but to $P_{\text {init }}$, which is $\sim 30 \%$ larger. As a consequence, its progenitor NS could be born with a mass $\sim 1.0 M_{\odot}$.

\section{Discussion and conclusions}

We have presented the modelling of the accretion-induced spinup phase undergone by two millisecond pulsars: the massive PSR J1614-2230 and the light PSR J0751+1807. In agreement with our previous work (Bejger et al. 2011), we showed that including the effect of the NS magnetic field is crucial for a correct understanding of the formation of millisecond pulsars. The dependence of our results on the prescriptions used for the magnetic field accretion-induced decay, on the estimate of the magnetic field, and on the EOS is remarkably small.

Our results indicate that the mass of the progenitor NS, born in a SNIb/c explosion, of PSR J1614-2230 was not lower than $1.9 M_{\odot}$ and therefore was very close to the currently measured value. This value is $\sim 0.2 M_{\odot}$ higher than the NS birth mass obtained in Tauris et al. $(2011,2012)$, and Lin et al. (2011), in which the accretion of $M_{\text {acc }}=0.3 M_{\odot}$ is required, while for our spin-up models, $\sim 0.06 M_{\odot}$ is sufficient. This large discrepancy partly comes from differences in the modelling of the spin-up phase but mostly from the fact that we do not model the whole evolution of the binary system.

Equation (14) in Tauris et al. (2012) yields a minimum value of $M_{\mathrm{acc}}$. This value is remarkably similar to our value of $M_{\mathrm{acc}}$. Our calculations show that this estimate is independent of the EOS and of the assumed model for the magnetic field and its accretion-induced decay. A reasonable estimate of the accreted 
mass could be obtained on the basis of the current parameters of the pulsar $(P, \dot{P}, M)$. In our approach the evolution depends on the mean accretion rate, which is not assumed to be the equilibrium one. However, there is a minimum value of the accreted mass $M_{\text {acc }}^{\min }$ needed to spin the star up to the observed rotational period. In our model, $M_{\mathrm{acc}}^{\min } \simeq 0.05 M_{\odot}$ (for details see Appendix A) and is obtained for a given $\tau_{\mathrm{acc}}^{\min }$ (see e.g. Figs. 4 and 7). The mean accretion rate is then given by $\dot{M}^{\text {min }}=M_{\text {acc }}^{\min } / \tau_{\text {acc }}^{\min }$. All these values for the amount of accreted matter are actually lower limits since processes in the NS magnetosphere and in the accretion disk at the origin of the ejection are not included.

In our approach (Bejger et al. 2011), relativistic effects are taken into account by introducing dimensionless function $f_{\mathrm{ms}}$ in Eq. (2). The role of GR for the radius $r_{0}$ is negligible, of the order of 1\% (for details see Appendix in Bejger et al. 2011). However, the specific angular momentum of a particle $l\left(r_{0}\right)$ calculated in the framework of GR is larger than obtained in Newtonian theory. The increase in $l$ due to GR is maximal at a marginally stable orbit (by a factor $\sqrt{2}$, see Kluźniak \& Wagoner 1985), and in cases considered in this paper $\left(r_{0}>r_{\mathrm{ms}}\right)$ is $\sim 10 \%$. As a consequence, disregarding GR effects results in less effective spin-up; the mass needed to reach given frequency is therefore higher by $\sim 10 \%$.

The progenitor of PSR J0751+1807 was itself born with a very low mass, which as we estimate, could be as low as $1.05 M_{\odot}$. Considering that the pulsar spun down after the recycling, the mass of the progenitor NS is lowered to $1.0 \mathrm{M}_{\odot}$.

The Roche lobe decoupling phase (RLDP), suggested recently by Tauris (2012) and Tauris et al. (2012) is related to some additional quasi-spherical accretion. Although we did not model the RLDP, we estimated the additional $M_{\text {acc }}$ (RLDP) as follows: When assuming a slow RLDP with a timescale of $\simeq 50 \mathrm{Myr}$ and a pre-RLDP accretion rate $\dot{M} \sim 10^{-10} M_{\odot} / \mathrm{yr}$ in the case of PSR J0751+1807, the upper limit on $M_{\text {acc }}(\mathrm{RLDP})$ is $0.005 M_{\odot}$. Because of the rapidly decreasing $\dot{M}$ and because some matter is ejected during the propeller phase, $M_{\text {acc }}(\mathrm{RLDP})$ is likely to be smaller. Considering that before the RLDP phase, a pulsar should have been spun up to a higher frequency than the one observed now, by accreting more mass before this phase, we can therefore conclude that the amount of matter accreted during the RLDP is negligible compared to the total mass accreted by the NS during the recycling process.

The wide range of NS birth masses, $1.0 M_{\odot}-1.9 M_{\odot}$ derived from our simulations, agrees with recent modellings by Ugliano et al. (2012) and Pejcha \& Thompson (2014) of supernova explosions of a large set of massive stars progenitors and metallicities in spherical symmetry.

Acknowledgements. We are grateful to Antonios Manousakis, Janusz Ziółkowski, Hans-Thomas Janka, and an anonymous referee for reading the manuscript and for helpful remarks and suggestions. We also acknowledge helpful remarks of participants of the CompStar 2011 Workshop (Catania, Italy, 9-12 May, 2011). This work was partially supported by the Polish NCN research grant no. 2013/11/B/ST9/04528 and by the COST Action MP1304 "NewCompStar".

\section{References}

Alpar, M. A., Cheng, A. F., Ruderman, M. A., \& Shaham, J. 1982, Nature, 300, 728

Antoniadis, J., Freire, P. C. C., Wex, N., et al. 2013, Science, 340, 448
Archibald, A. M., Stairs, I. H., Ransom, S. M., et al. 2009, Science, 324, 1411

Bassa, C. G., van Kerkwijk, M. H., \& Kulkarni, S. R. 2006, A\&A, 450, 295

Bassa, C. G., Patruno, A., Hessels, J. W. T., et al. 2014, MNRAS, 441, 1825

Bednarek, I., Haensel, P., Zdunik, J. L., Bejger, M., \& Mańka, R. 2012, A\&A, 543, A157

Bejger, M. 2013, A\&A, 552, A59

Bejger, M., Zdunik, J. L., \& Haensel, P. 2010, A\&A, 520, A16

Bejger, M., Fortin, M., Haensel, P., \& Zdunik, J. L. 2011, A\&A, 536, A87

Bejger, M., Fortin, M., Haensel, P., \& Zdunik, J. L. 2013, in Feeding Compact Objects on All Scales, Proc. IAU Symp., 290, 109

Bhalerao, V. B., \& Kulkarni, S. R. 2011, ApJ, 737, L1

Bisnovatyi-Kogan, G. S., \& Komberg, B. V. 1971, Soviet. Astron, 18, 217

Chabrier, G., Brassard, P., Fontaine, G., \& Saumon, D. 2000, ApJ, 543, 2016

Colpi, M., Possenti, A., Popov, S., \& Pizzolato F. 2001, in Physics of neutron star interiors, eds. D. Blaschke, A. Sedrakian, \& N. K. Glendenning (Springer), Lect. Notes Phys., 578, 440

Cumming, A., Zweibel, E., \& Bildsten, L. 2001, ApJ, 557, 958

Demorest, P. B., Pennucci, T., Ransom, S. M., Roberts, M. S. E., \& Hessels, J. W. T. 2010, Nature, 467, 1081

Douchin, F., \& Haensel, P. 2001, A\&A, 380, 151

Drago, A., Lavagno, A., \& Pagliara, G. 2014a, Phys. Rev. D, 89, 043014

Drago, A., Lavagno, A., \& Pagliara, G. 2014b, Phys. Rev. C, 90, 065809

Fortin, M. 2012, Ph.D. Thesis, http://tel.archives-ouvertes.fr/ tel-00731478

Francischelli, G. J., Wijers, R. A. M. J., \& Brown, G. E. 2002, ApJ, 565, 471

Freire, P. C. C., Bassa, C. G., \& Wex, N., et al. 2011, MNRAS, 412, 2763

Geppert, U., \& Urpin, V. 1994, MNRAS, 271, 490

Glendenning, N. 1985, ApJ, 293, 470

Haensel, P., Potekhin, A. Y., \& Yakovlev, D. G. 2007, Neutron Stars 1, Equation of State and Structure (New York: Springer)

Hansen, B. M. S, \& Phinney, E. S. 1998, MNRAS, 294, 557

Hessels, J. W. T., Ransom, S. M., Stairs, I. H., et al. 2006, Science, 311, 1901

Istrate, A. G., Tauris, T. M., \& Langer, N. 2014, A\&A, 571, A45

Kiel, P. D., Hurley, J. R., Bailes, M., \& Murray, J. R. 2008, MNRAS, 388, 393

Kiziltan, B., \& Thorsett, S. E. 2010, ApJ, 715, 335

Kluźniak, W., \& Rappaport, S. 2007, ApJ, 671, 1990

Kluźniak, W., \& Wagoner, R. 1985, ApJ, 548, 297

Langer, N., Deutschmenn, A., Wellstein, S., \& Hoeflich, P. 2000, A\&A, 362, 1046

Lin, J., Rappaport, S., Podsiadlowski, P., et al. 2011, ApJ, 732, 70

de Loore, C. W. H., \& Doom, C. 1993, Structure and Evolution of Single and Binary Stars (Dordrecht: Kluwer Academic Publishers)

Lorimer, D. R. 2008, Liv. Rev. Relativ., 11, 8

Manchester, R. N., Hobbs, G. B., Teoh, A., \& Hobbs, M. 2005, AJ, 129, 1993

Nandy, A. 1974, ApJ, 190, 385

Nice, D. J. et al., 2008, in 40 Years of Pulsars: Millisecond Pulsars, Magnetars and More, AIP Conf. Proc., 983, 453

Osłowski, S., Bulik, T., Gondek-Rosińska, D., \& Belczyński, K. 2011, MNRAS, 413, 461

Papitto, A., Ferrigno, C., Bozzo, E., et al. 2013, Nature, 501, 517

Patruno, A., Archibald, A. M., Hessels, J. W. T., et al. 2014, ApJ, 781, L3

Pejcha, O., \& Thompson, T. A. 2015, ApJ, 801, 90

Radhakrishnan, V., \& Srinivasan, G. 1982, Current Sci., 51, 1096

Romani, R. W. 1990, Nature, 347, 741

Sawyer, R. F. 1972, ApJ, 176, 205; Erratum, 178, 279

Shibazaki, N., Muarakami, T., Shaham, J., \& Nomoto, K. 1989, Nature, 342, 656

Shklovskii, I. S. 1980, Sov. Astron., 13, 562

Spitkovsky, A. 2006, ApJ, 648, L51

Taam, R. E., \& van den Heuvel, E. P. J. 1986, ApJ, 305, 235

Tauris, T. M. 2012, Science, 335, 561

Tauris, T. M., \& van den Heuvel, E. P. J. 2006, in Compact stellar X-ray sources (CUP), 623

Tauris, T. M., Langer, N., \& Kramer, M. 2011, MNRAS, 416, 2130

Tauris, T. M., Langer, N., \& Kramer, M. 2012, MNRAS, 425, 1601

Ugliano, M., Janka, H.-T., Marek, A., \& Arcones, A. 2012, ApJ, 757, 69

van den Heuvel, E. P. J., \& Bitzaraki, O. 1995, A\&A, 297, L41

Wijers, R. A. M. J. 1997, MNRAS, 287, 607

Wijnands, R., \& van der Klis, M. 1998, Nature, 394, 344

Zhang, C. M., \& Kojima, Y. 2006, MNRAS, 366, 137 


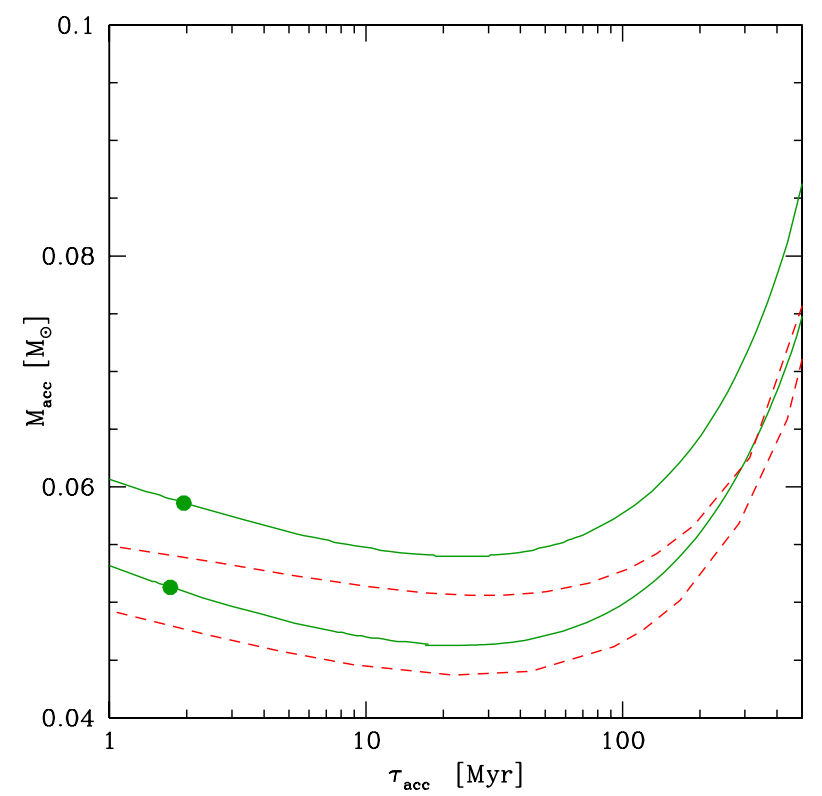

Fig. A.1. Mass of accreted matter as a function of the time needed to spin-up the progenitor NS to the observed state (i.e. $M, B$, and $f$ ) for PSR J1614-2230 (upper curves) and PSR J0751+1807. Exact results solid (green) curves; approximation - dashed (red) lines.

\section{Appendix A: Approximate solution}

The properties of the solutions of Eqs. (1) and (2) allow us to determine an approximate solution for our model. It contains only the parameters of the final configuration: $B, f, M$ and a given mean accretion rate $\dot{M}$ and enables the properties of the pre-accretion NS to be estimated. The spin-up Eq. (1) can be factorized as

$$
\frac{\mathrm{d} J}{\mathrm{~d} M_{\mathrm{b}}}=\sqrt{G M r_{\mathrm{c}}} \cdot \lambda(f),
$$

where $\lambda \equiv l_{\text {tot }} / \sqrt{G M r_{\mathrm{c}}}$ is the ratio of total specific angular momentum of an accreted particle to its value at the corotation radius. The main dependence on rotational frequency is included in $\sqrt{r_{\mathrm{c}}} \sim f^{-1 / 3}$.

The ratios of the characteristic lengths describing the system $\left(r_{\mathrm{m}}, r_{\mathrm{c}}, r_{0}\right)$ do not change significantly along a spin-up track, except for a small region of very slow rotation. Therefore, one can assume that $\lambda(f)$ is constant along spin-up track as the Eq. (2) depends on $r_{\mathrm{m}} / r_{0}$ and $r_{\mathrm{c}} / r_{0}$. This assumption holds with an accuracy of about $20 \%$. However, $\lambda$ is a rather sensitive function of the mean accretion rate. For example, for the set of evolutionary tracks with different accretion rates presented in Figs. 1 and 2, the value of $\lambda$ changes by more than one order of magnitude.
Neglecting the change in the NS moment of inertia I (which is a good assumption for configurations close to the maximum mass and/or for relatively small amount of accreted mass), we obtain the formula

$\Delta M \simeq \frac{3}{4} \frac{J_{f}}{l_{f}}, \quad$ with $\quad l_{f}=l\left(r_{0 f}\right)-l_{\mathrm{m}}$,

where $J_{f}$ is the angular momentum, $l\left(r_{0 f}\right)$ is specific angular momentum of an accreted particle, and $l_{\mathrm{m}}$ is magnetic torque divided by $\dot{M}$, all three quantities corresponding to the final (presently observed) state of the pulsar.

Since the magnetic torque is proportional to $1 / \dot{M}=\tau / \Delta M$ in the limit of a low accretion rate (large $\tau$ ), the angular momentum transferred to the star is $l_{f}=l\left(r_{0}\right) \cdot(1-A / \dot{M})=$ $l\left(r_{0}\right) \cdot(1-A \tau / \Delta M)$, the dependence on $\tau$ is almost linear, as shown in Figs. 4 and 7 :

$\Delta M \simeq \frac{3}{4} \frac{J_{f}}{l_{0}}+A \cdot \tau, \quad$ with $\quad A=\frac{\mu^{2}}{9 r_{0}^{3} l\left(r_{0}\right)}\left(3-2 \sqrt{\frac{r_{\mathrm{c}}^{3}}{r_{0}^{3}}}\right)$.

A simple estimate of the amount of accreted matter needed to spin up a NS to a given configuration can be obtained from Eq. (A.3). The radius $r_{0 f}$ at the inner boundary of the disk can be calculated by solving the simple algebraic equation Eq. (2) for values of $f, B$, and $M$ corresponding the ones of the final configuration. Then $l\left(r_{0 f}\right)$ can be determined by the analytic formula given in Bejger et al. (2010). Examplary results based only on the present-day parameters are shown in Fig. A.1. For a given duration of the accretion phase, one can then derive the amount of matter accreted by the NS to reach its current configuration. Then the birth mass can be simply derived and the pre-accretion magnetic field is given by Eq. (6).

For high $\dot{M}$, the value of $A$ depends sensitively on the solution of Eq. (2), and the function $A(\dot{M})$ changes its sign at the point $\dot{M}^{\text {min }}$ corresponding to

$\left(\frac{r_{0}}{r_{\mathrm{c}}}\right)^{3 / 2}=\frac{2}{3}, \quad$ i.e., $\quad r_{\mathrm{c}}=1.31 r_{0}$.

For $\dot{M}>\dot{M}^{\text {min }}$, the accreted mass $\Delta M$ is a decreasing function of $\tau_{\text {acc }}$ (see inserts in Figs. 4 and 7). In our case, $\dot{M}^{\text {min }} \simeq 2 \times$ $10^{-9} M_{\odot} / \mathrm{yr}$ and thus is more than one order of magnitude lower than the Eddington limit $\simeq 3 \times 10^{-8} M_{\odot} / \mathrm{yr}$. The minimum mass needed to spin a pulsar up to its observed frequency depends on the value of the specific angular momentum of a particle at the corotation radius and is given by $\Delta M^{\mathrm{min}}=0.75 J_{f} / l\left(0.763 r_{\mathrm{c}}\right)$. This quantity does not depend on the accretion rate. 